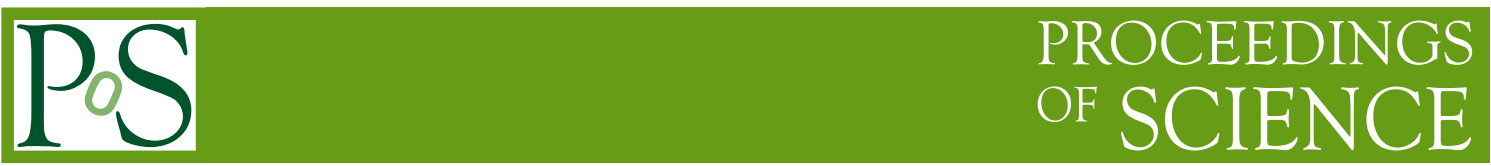

\title{
COMPASS results on the Collins and Sivers asymmetries
}

\author{
Anna Martin* ${ }^{\dagger}$ \\ Trieste University and INFN \\ E-mail: anna.martinets.infn.it
}

The investigation of transverse spin and transverse momentum effects is an important part of the physics programme of COMPASS, a fixed target experiment at the CERN SPS taking data since 2002. This experimental program is carried on by measuring semi-inclusive deep inelastic scattering (SIDIS) of high energy muons off different nucleon targets, both unpolarised or polarised. By measuring the asymmetries in the azimuthal distributions of the final state hadrons it is possible to access the new parton distribution functions which encode the correlations among the transverse spin and transverse momentum of the partons, and the spin of the parent nucleon. Among the different leading order azimuthal asymmetries, all measured in COMPASS, the so-called Collins and Sivers asymmetries are of particular interest being related to the transversity and Sivers functions respectively. The Collins and the Sivers asymmetries have been measured in COMPASS using first a transversely polarised deuteron target and, more recently, a transversely polarised proton target finding clear evidence for non zero asymmetries. The results are reviewed in this contribution, which is moslty dedicated to the more recent results, namely the Collins and Sivers asymmetries on proton for identified charged hadrons.

XXI International Workshop on Deep-Inelastic Scattering and Related Subject -DIS2013,

22-26 April 2013

Marseilles, France

* Speaker.

${ }^{\dagger}$ on behalf of the COMPASS Collaboration 
The SIDIS process is a unique tool to investigate the transverse structure of the nucleon. It allows to perform precise measurements of observables which have a clear interpretation in the recently developed QCD framework, in which the parton distribution functions (PDFs) do not depend only on $x$ and $Q^{2}$ but also on the transverse momentum of the parton [1]. By measuring the amplitudes of the different modulations in the azimuthal distributions of the SIDIS final state hadrons, it is in fact possible to disentangle the effects due to the different transverse momentum dependent (TMD) PDFs. Also, using deuteron (neutron) and proton targets, and by identifying the final state hadrons, it is possible to gwt information on the contributions of quarks with different flavours. The most popular among these azimuthal distributions are the Collins and the Sivers asymmetries which are measured using transversely polarised nucleon targets and which give access to the transversity and the Sivers PDFs.

The relevance of the transversity PDF, which gives the correlation between the transverse polarisation of quarks inside a transversely polarised nucleon and the parent nucleon polarisation, is today very well known. Together with the unpolarised distribution and the helicity distribution, transversity is needed to completely describe of the nucleon structure at leading order in the collinear approximation. Also, these three PDFs are the only ones surviving under integration over the intrinsic transverse momentum. Transversity is still poorly known since, being chiral-odd, it can not be accessed in inclusive DIS. However it can be extracted from the Collins asymmetry measured in SIDIS, where it appears convoluted with the chiral-odd Collins fragmentation function. Independent information on this new fragmentation function, and clear evidence that it is different from zero, came recently from the Belle and Babar measurements of the $e^{+} e^{-} \rightarrow$ hadrons process.

The Sivers function is one of the most famous TMD PDFs and describes the correlation between the parton transverse momentum and the transverse spin of the parent nucleon. It is "T-odd" and can be different from zero thanks to final (or initial) state interactions. In SIDIS this function can be extracted from the so-called Sivers asymmetry which is proportional to the convolution over transverse momenta of the Sivers function and the usual spin averaged fragmentation function. It can be measured also in Drell-Yan processes, where it is expected to have opposite sign with respect to SIDIS.

The first experimental evidence for these transverse spin effects came in 2005, when the first results on SIDIS off transversely polarised targets where published by the HERMES and COMPASS experiments. Since then a lot of progress has been done, and COMPASS gave and is giving an important contribution in this field, as summarised in the following.

COMPASS is a fixed target experiment at the CERN SPS with a wide physics programme. The two-stages spectrometer has been designed to cope with high beam intensities and to have a large and as uniform as possible acceptance for the final state hadrons down to $1.5 \mathrm{GeV} / \mathrm{c}$ momentum. Charged hadrons are identified by a RICH detector, and final state muon identification is also performed. For the SIDIS measurements, the incoming muon momentum is measured event by event, and a complex cryogenic system allows to use frozen spin targets, $120 \mathrm{~cm}$ long, which can be polarised either longitudinally or transversely with respect to the beam direction. The spectrometer was upgraded in 2005 when the central region of the RICH detector was changed and a new polarised target magnet with a larger angular acceptance was installed. SIDIS data with a $160 \mathrm{GeV}$ $\mu^{+}$beam and with the transversely polarised deuteron target $\left({ }^{6} \mathrm{LiD}\right)$ were taken in the years 2002 

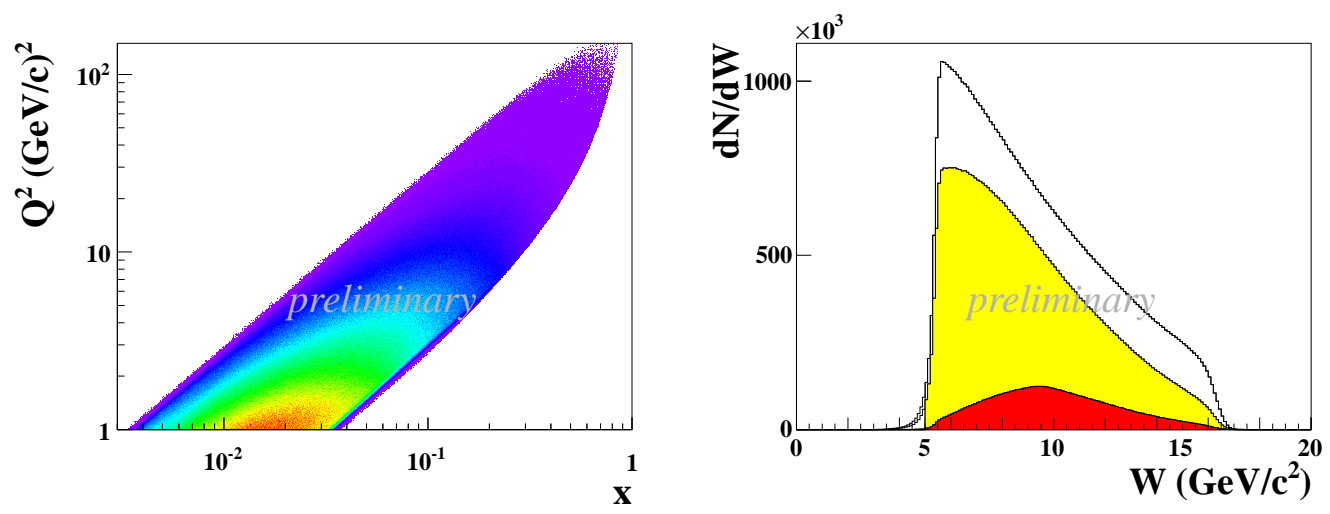

Figure 1: Left: $x-Q^{2}$ correlation for charged pions. Right: $W$ distribution for charged hadrons (upper histogram), pions (middle) and kaons (lower).

to 2004 . In 2007 and 2010 the transversely polarised proton target $\left(\mathrm{NH}_{3}\right)$ was used, again with the $160 \mathrm{GeV} \mu^{+}$beam.

The data analysis is very similar for all the years of data taking and the relevant cuts applied to select the "standard sample" are also the same. Only events with photon virtuality $Q^{2}>1$ $\mathrm{GeV}^{2}$, fractional energy of the virtual photon $0.1<y<0.9$, and mass of the hadronic final state system $W>5 \mathrm{GeV} / \mathrm{c}^{2}$ are considered. The charged hadrons are required to have at least $0.1 \mathrm{GeV} / \mathrm{c}$ transverse momentum $p_{T}^{h}$ with respect to the virtual photon direction and a fraction of the available energy $z>0.2$. No momentum cut is applied to measure the asymmetries for charged hadrons. Charged pions and kaons are identified with negligible contamination in the momentum range from Cherenkov threshold $\left(2.7 \mathrm{GeV} / \mathrm{c}\right.$ and $9.7 \mathrm{GeV} / \mathrm{c}$ respectively) up to $50 \mathrm{GeV} / \mathrm{c}$. The $x-Q^{2}$ correlation for charged pions from 2010 data is shown in fig. 1 (left). As can be seen, the $x$ range goes from $x \simeq 3 \cdot 10^{-3}$ to $x \simeq 0.7$ with relatively large $Q^{2}$ values in the valence region. Figure 1 (right) gives the $W$ distribution for charged hadrons, pions and kaons. In the standard analysis, the transverse-spin asymmetries are measured separately for positive and negative hadrons (or pions or kaons) as functions of $x, z$ or $p_{T}^{h}$. The complete definition (namely sign and kinematic factors) of the asymmetries can be found in the published papers $[2,3]$.

The Collins and Sivers asymmetries for positive and negative hadrons from the 2004 deuteron data $[4,5]$ turned out to be compatible with zero within the few percent uncertainties, at variance with the non-zero results obtained by the HERMES experiment on proton [6,7]. Similar results were obtained for charged pions and kaons [8]. These data could be understood in terms of cancellation between the $u$ and $d$ quark contributions in the deuteron target, and were used in global fits to extract the transversity and Sivers functions. Still today these COMPASS data are the only SIDIS data collected with a transversely polarised deuteron target. Recently, the Collins and the Sivers asymmetries have been measured with a ${ }^{3} \mathrm{He}$ target at JLab [9], but at much lower energy. Further measurements on deuteron at COMPASS could also be performed in the future, if such measurements will be supported by a strong physics case.

Measurements with transversely polarised proton targets at COMPASS were warmly expected by the international community, to get information on the $Q^{2}$ dependence of these effects and thus 

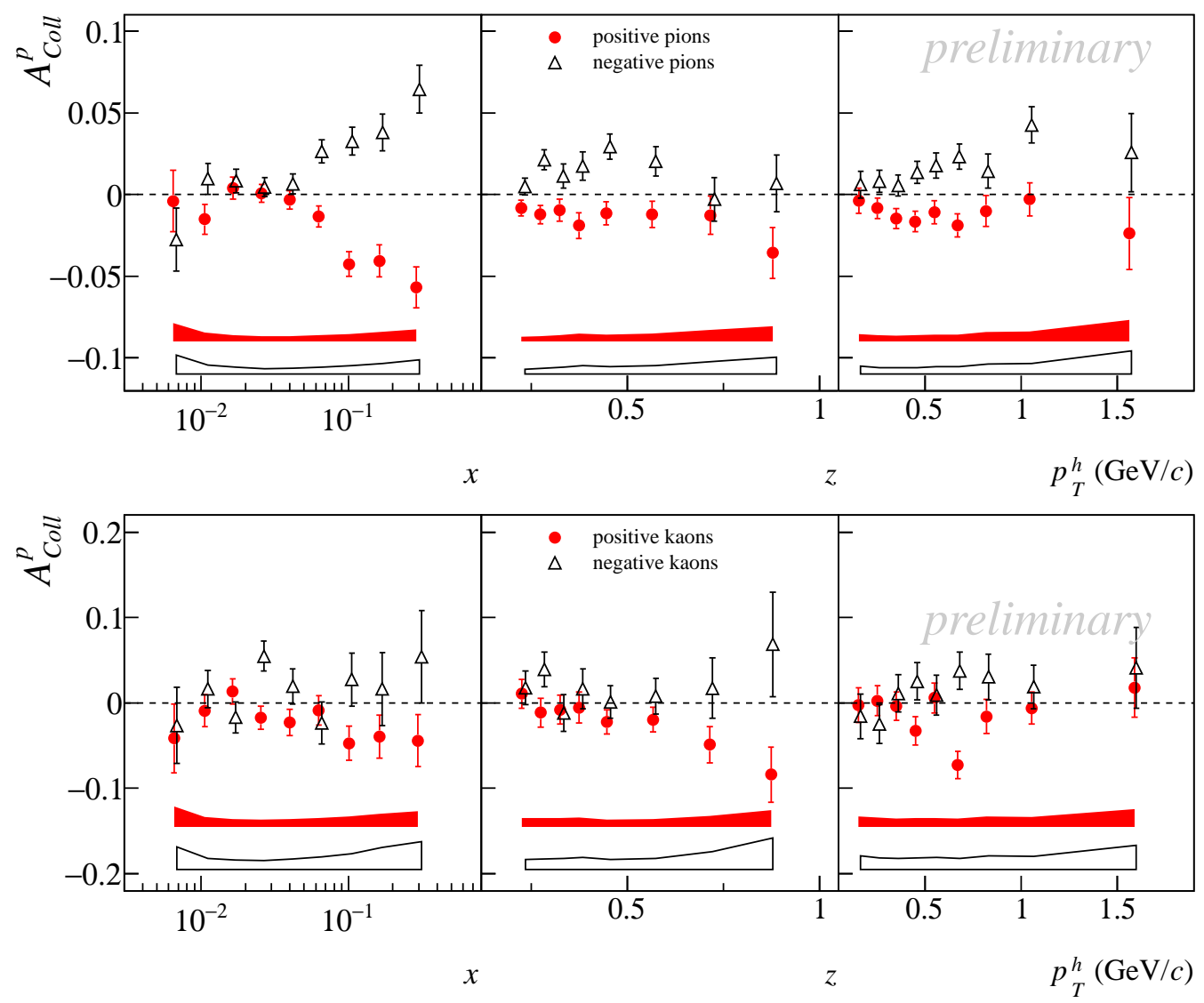

Figure 2: Collins asymmetries for positive (open points) and negative (closed points) pions (upper panel) and kaons (lower panel) from the 2010 COMPASS proton data.

on the evolution of the TMD PDFs. The first results for the charged hadrons Collins and Sivers asymmetries on proton from COMPASS [10] came from the analysis of the 2007 data. They have been recently confirmed by the higher precision results (statistical and point-to-point systematic uncertainties about twice smaller and negligible systematic scale uncertainties) obtained from the 2010 data $[2,3]$.

Very recently, the results for charged pions and kaons have also been produced [11] and they are discussed in the following.

The Collins asymmetries from the 2010 data for charged $\pi$ and $\mathrm{K}$ are shown in fig. 2. As expected, the values of the pion asymmetries are very close to those of the charged hadron asymmetries. The pion asymmetries are compatible with zero in the previously unmeasured $x<0.03$ region while at larger $x$ they are clearly different from zero, with opposite sign for positive and negative hadrons and in nice agreement, both in sign and in magnitude, with the HERMES results [6]. There is no indication for lower values of the Collins asymmetry at the higher COMPASS $Q^{2}$ values as compared to the HERMES measurement. For the charged $\mathrm{K}$, the statistical uncertainties are large, still the data give some interesting indication. In particular there is some indication for values different from zero and with the same sign as in the pion case, at variance with the HERMES 

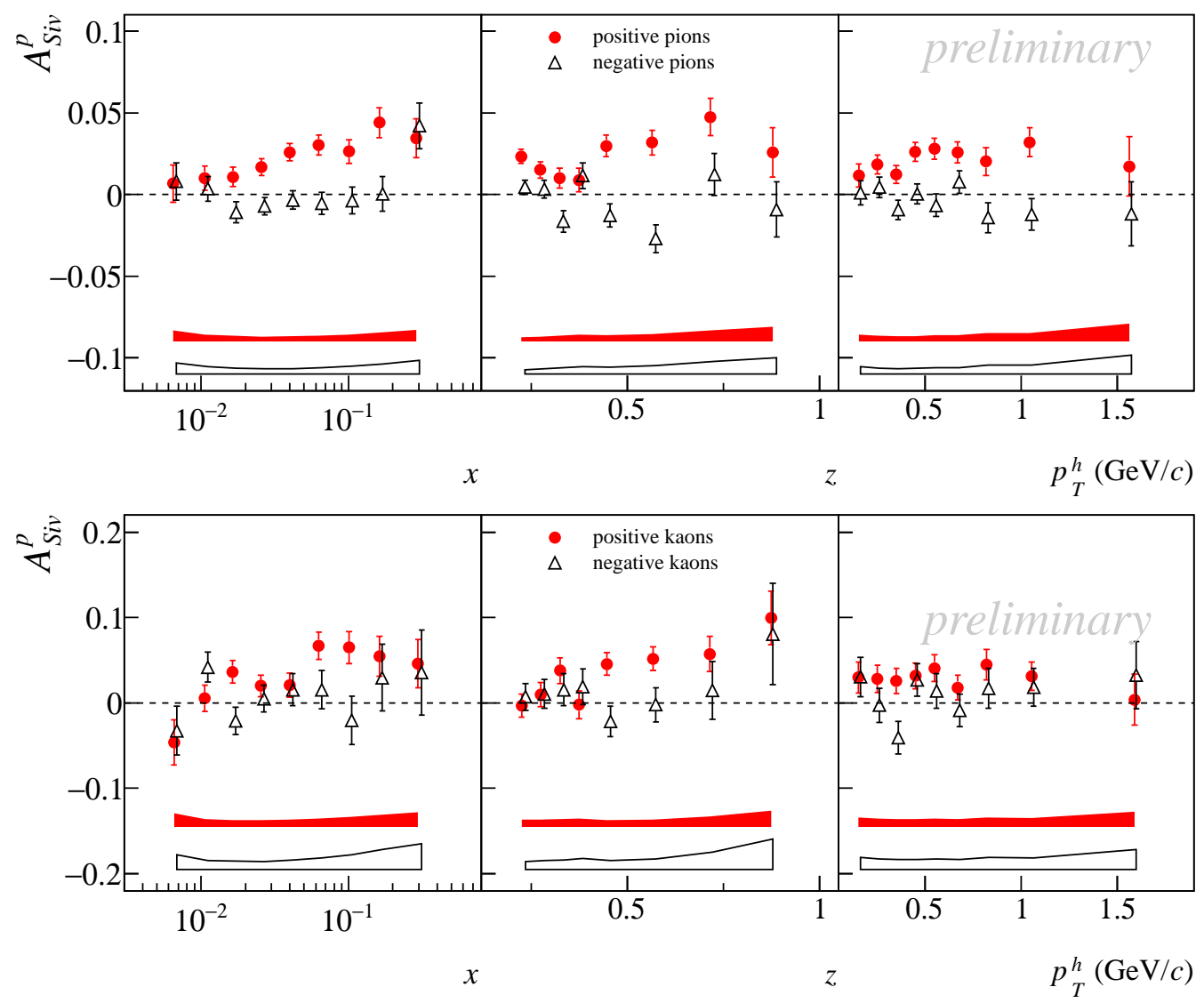

Figure 3: Sivers asymmetries for positive (open points) and negative (closed points) pions (upper panel) and kaons (lower panel) from the 2010 COMPASS proton data.

results.

The Sivers asymmetries for charged $\pi$ and $\mathrm{K}$ are given in fig. 3. Again, the pion asymmetries are very close to those for charged hadrons. In particular, for $\pi^{-}$they are compatible with zero with some indication for small negative values over the entire $x$ range but in the last bin. In the case of $\pi^{+}$, the Sivers asymmetry is positive down to very small $x$ values and in the $x>0.03$ region it is smaller than the same asymmetry measured by HERMES [7], a fact which can be understood in terms of the recent calculations on TMDs evolution. In the case of $\mathrm{K}^{+}$, the Sivers asymmetry is clearly positive, again smaller than that measured by HERMES, but higher than the one measured in COMPASS for $\pi^{+}$. A larger Sivers asymmetry for positive kaons was already observed by HERMES and it will be interesting to see the impact of the new COMPASS result.

It must be noted that when these asymmetries are used in global analysis, the statistical correlations between the asymmetries measured as functions of $x, z$ or $p_{T}^{h}$ should be taken into account. They have been measured from the data [2] and are all available. As an example fig. 4 shows the correlation coefficients in the different $x, z$ bins in the case of charged hadrons.

Summarising, precise measurements of the Collins and Sivers asymmetries on proton have been recently produced by COMPASS both for non-identified and identified final state charged 


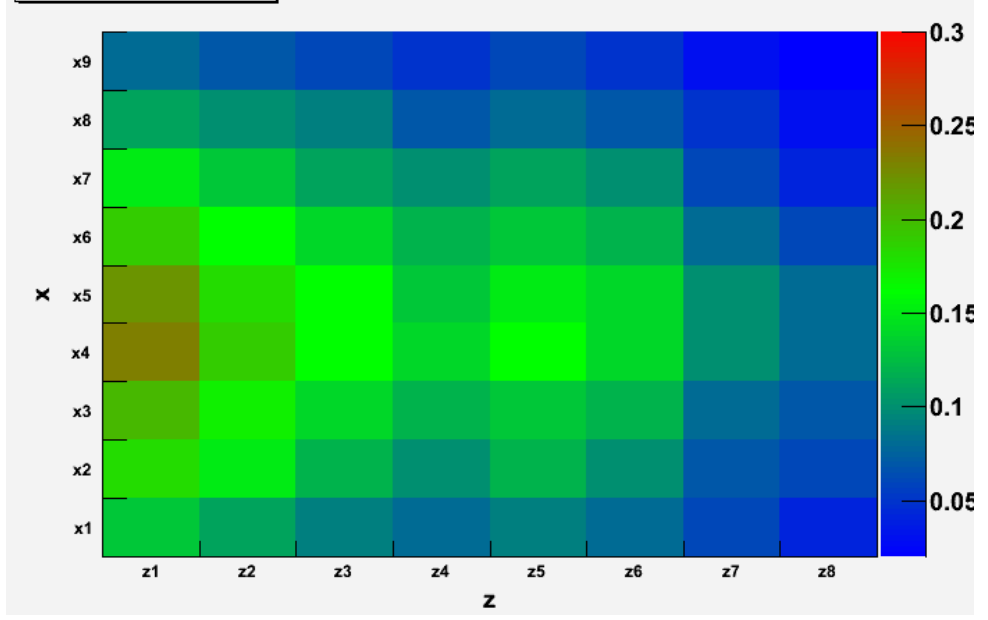

Figure 4: Correlation coefficients between the asymmetries for charged hadrons measured as function of $x$ and $z$.

hadrons. While no signal was observed in the previous measurements with a deuteron target, clear non-zero signals are seen for the Collins and the Sivers asymmetries obtained from the proton data. These results, when used together with the results at lower beam energy produced by HERMES and the past and future Jefferson Lab experiment, will give key input for testing the $Q^{2}$ evolution and more accurate extractions of the transversity and Sivers PDFs. More results for the Collins and Sivers asymmetries will come soon from COMPASS. In particular, a multidimensional analysis to better disentangle the different effects is planned. On a longer term, the possibility of performing further measurements with transversely polarised targets is under investigation.

\section{References}

[1] C. A. Aidala, S. D. Bass, D. Hasch and G. K. Mallot, Rev. Mod. Phys. 85 (2013); 655 V. Barone, F. Bradamante and A. Martin, Prog. Part. Nucl. Phys. 65 (2010) 267.

[2] C. Adolph et al. [COMPASS Collaboration], Phys. Lett. B 717 (2012) 376.

[3] C. Adolph et al. [COMPASS Collaboration], Phys. Lett. B 717 (2012) 383.

[4] V. Y. .Alexakhin et al. [COMPASS Collaboration], Phys. Rev. Lett. 94 (2005) 202002.

[5] E. S. Ageev et al. [COMPASS Collaboration], Nucl. Phys. B 765 (2007) 31.

[6] A. Airapetian et al. [HERMES Collaboration], Phys. Lett. B 693 (2010) 11.

[7] A. Airapetian et al. [HERMES Collaboration], Phys. Rev. Lett. 103 (2009) 152002.

[8] M. Alekseev et al. [COMPASS Collaboration], Phys. Lett. B 673 (2009) 127.

[9] X. Qian et al. [Jefferson Lab Hall A Collaboration], Phys. Rev. Lett. 107 (2011) 072003.

[10] M. G. Alekseev et al. [COMPASS Collaboration], Phys. Lett. B 692 (2010) 240.

[11] A. Martin [ COMPASS Collaboration], SPIN2012 Proceedings, arXiv:1303.2076 [hep-ex]. 